\title{
IGR J17361-4441: a possible new accreting X-ray binary in NGC 6388
}

\author{
E. Bozzo ${ }^{1}$, C. Ferrigno ${ }^{1}$, J. Stevens ${ }^{2}$, T. M. Belloni ${ }^{3}$, J. Rodriguez ${ }^{4}$, P. R. den Hartog ${ }^{5}$, A. Papitto ${ }^{6}$, \\ I. Kreykenbohm ${ }^{7}$, F. Fontani ${ }^{8}$, and L. Gibaud ${ }^{1}$
}

\author{
${ }^{1}$ ISDC Data Centre for Astrophysics, Chemin dEcogia 16, 1290 Versoix, Switzerland \\ e-mail: enrico.bozzo@unige.ch \\ 2 CSIRO Astronomy \& Space Science, Australia Telescope National Facility, PO Box 76, Epping, NSW 1710, Australia \\ 3 INAF, Osservatorio Astronomico di Brera, via E. Bianchi 46, 23807 Merate (LC), Italy \\ ${ }^{4}$ Laboratoire AIM, CEA/IRFU - CNRS/INSU - Université Paris Diderot, CEA DSM/IRFU/SAp, 91191 Gif-sur-Yvette, France \\ 5 Stanford University HEPL/KIPAC Physics, 382 via Pueblo Mall Stanford, 94305, USA \\ ${ }^{6}$ Dipartimento di Fisica, Universitá degli Studi di Cagliari, SP Monserrato-Sestu, KM 0.7, 09042 Monserrato, Italy \\ 7 Dr. Karl Remeis-Sternwarte \& Erlangen Centre for Astroparticel Physics, Sternwartstr. 7, 96049 Bamberg, Germany \\ 8 INAF - Osservatorio Astrofisico di Arcetri, L.go E. Fermi 5, 50125 Firenze, Italy \\ Received 5 September 2011 / Accepted 22 September 2011
}

\section{ABSTRACT}

\begin{abstract}
IGR J17361-4441 is a newly discovered INTEGRAL hard X-ray transient, located in the globular cluster NGC 6388. We report here the results of the X-ray and radio observations performed with Swift, INTEGRAL, RXTE, and the Australia Telescope Compact Array (ATCA) after the discovery of the source on 2011 August 11. In the X-ray domain, IGR J17361-4441 showed virtually constant flux and spectral parameters up to 18 days from the onset of the outburst. The broad-band $(0.5-100 \mathrm{keV})$ spectrum of the source could be reasonably well described by using an absorbed power-law component with a high energy cut-off $\left(N_{\mathrm{H}} \simeq 0.8 \times 10^{22} \mathrm{~cm}^{-2}, \Gamma \simeq 0.7-\right.$ 1.0 , and $E_{\text {cut }} \simeq 25 \mathrm{keV}$ ) and displayed some evidence of a soft component below $\sim 2 \mathrm{keV}$. No coherent timing features were found in the RXTE data. The ATCA observation did not detect significant radio emission from IGR J17361-4441, and provided the most stringent upper limit (rms $14.1 \mu \mathrm{Jy}$ at $5.5 \mathrm{GHz}$ ) to date on the presence of any radio source close to the NGC 6388 center of gravity. The improved position of IGR J17361-4441 in outburst determined from a recent target of opportunity observation with Chandra, together with the X-ray flux and radio upper limits measured in the direction of the source, argue against its association with the putative intermediate-mass black hole residing in the globular cluster and with the general hypothesis that the INTEGRAL source is a black hole candidate. IGR J17361-4441 might be more likely a new X-ray binary hosting an accreting neutron star. The ATCA radio non-detection also permits us to derive an upper limit to the mass of the suspected intermediate massive black hole in NGC 6388 of $\lesssim 600 M_{\odot}$. This is a factor of 2.5 lower than the limit reported previously.
\end{abstract}

Key words. X-rays: binaries - X-rays: stars - X-rays: individuals: IGR J17361-4441

\section{Introduction}

IGR J17361-4441 was discovered by the IBIS/ISGRI telescope (Ubertini et al. 2003) onboard INTEGRAL (Winkler et al. 2003) on 2011 August 11 (Gibaud et al. 2011). The source was located in the direction of the globular cluster (GC) NGC 6388 and detected at an X-ray flux of $9.7 \times 10^{-11} \mathrm{erg} \mathrm{cm}^{-2} \mathrm{~s}^{-1}(20-$ $100 \mathrm{keV}$ ). Follow-up observations carried out with Swift and Chandra (Ferrigno et al. 2011b; Pooley et al. 2011) provided a first description of the source X-ray emission in the soft energy band $(0.3-10 \mathrm{keV})$, a refined source position at RA = 17:36:17.418, Dec = -44:44:05.98 (J2000; the nominal Chandra positional accuracy is 0.6 arcsec, $90 \%$ c.l.) and confirmed its localization in NGC 6388. In the past, several X-ray observational campaigns have been carried out in the direction of this GC, as it is believed to host an intermediate-mass black hole (IMBH) at its center of gravity (hereafter COG; Lanzoni et al. 2007). Here we report on the analysis of all the available X-ray data collected during the first 18 days of the outburst observed from IGR J17361-4441, and use a simultaneous observation in the radio domain with the ATCA telescope to investigate the nature of the source.

\section{Data analysis}

\subsection{INTEGRAL}

We analyzed all the available data for the IBIS/ISGRI (20$150 \mathrm{keV}$, Lebrun et al. 2003) and for the JEM-X telescope (5$20 \mathrm{keV}$, Lund et al. 2003) onboard INTEGRAL that were performed in the direction of IGR J17361-4441 from 2011 August 11 (rev. 1078) to 2011 August 29 (rev. 1083). Data analysis was carried out by using version 9.0 of the OSA software distributed by the ISDC (Courvoisier et al. 2003). In Fig. 1, we show the IBIS/ISGRI mosaicked significance map around IGR J173614441 in the 20-100 keV energy band (total exposure time $\sim 500 \mathrm{ks}$ ). In this mosaic, IGR J17361-4441 is detected at a significance level of $15 \sigma$ at an average flux of $1.2 \times 10^{-10} \mathrm{erg} / \mathrm{cm}^{2} / \mathrm{s}$. The corresponding IBIS/ISGRI spectrum is discussed later in Sect. 2.3. From rev. 1078 to 1083, IGR J17361-4441 was inside the field of view (FOV) of the X-ray monitor onboard INTEGRAL, JEM-X, only for a total effective exposure time of $17 \mathrm{ks}$ and was not detected. We derived a $3 \sigma$ upper limit to the 3-35 keV X-ray flux from IGR J17361-4441 of $3.8 \mathrm{mCrab}^{1}$

1 Calculated by using the calibration observation of the Crab in satellite rev. 967 (from 2010 September 13 to 15). 


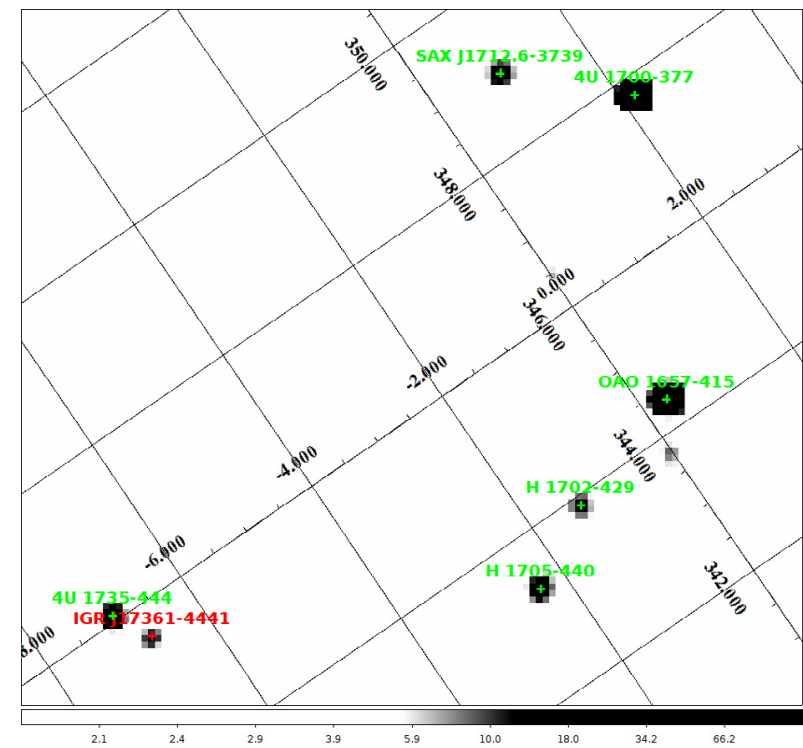

Fig. 1. IBIS/ISGRI FOV around IGR J17361-4441 (20-100 keV energy band). We used data from rev. 1078 to 1083 . The horizontal band on the bottom of the image shows the colour-coded significances in the mosaic expressed in units of $\sigma$.

(corresponding to $1.1 \times 10^{-10} \mathrm{erg} / \mathrm{cm}^{2} / \mathrm{s}$ ). This is compatible with the value expected based on the broad-band spectral properties of the source (see Sect. 2.3).

\subsection{RXTE}

A follow-up observation of IGR J17361-4441 was performed with the PCA onboard RXTE (Bradt et al. 1993) on 2011 August 17 (total exposure time $1.6 \mathrm{ks}$ ). The pointing was carried out with an offset of $\sim 0.53$ degrees from the nominal position of IGR J17361-4441 to limit ${ }^{2}$ the contamination from the bright nearby low mass X-ray binary $4 \mathrm{U} 1735-444$. The good time intervals were based on a maximum offset from the source of 0.55 degrees, a minimum elevation of ten degrees over the Earth limb, an electron ratio lower than 0.2, and at least ten minutes having passed since the SAA passage (see also, e.g. Ferrigno et al. 2011a). We analyzed the PCA event mode data in the GoodXenon configuration, which has a time resolution of $0.96 \mu$ s and selected all events from the PCU 1, 2, and 4 (PCU0 suffered a breakdown during the observation). The event arrival times were all barycenter-corrected to the Solar System centerof-mass using the available tool FAXBARY and the most-accurately determined source position in Chandra data (see Sect. 1). We constructed a power density spectrum after accumulating light curves in bins of $0.49 \mathrm{~ms}$, so that frequencies of up to $\sim 1 \mathrm{kHz}$ could be explored when searching for coherent signals (see Fig. 2). To maximize the frequency resolution, we used a single interval to compute the fast Fourier transform (FFT). We did not find any significant peak at a $3 \sigma$ c.l. (we followed the approach described in Vaughan et al. 1994). The high background level of the PCA observation and the contamination from 4U 1735-444 did not permit an accurate estimation of the corresponding pulsed fraction upper limit.

\footnotetext{
2 By using archival PCA observations, we estimated an average onaxis count-rate over three active PCUs of $\sim 1400 \mathrm{cts} / \mathrm{s}$ for $4 \mathrm{U} 1735-444$. Therefore, this source might contribute up to $\sim 25 \%$ of the signal from the observation of IGR J17361-4441, which has a backgroundsubtracted count-rate of $\sim 10 \mathrm{cts} / \mathrm{s}$.
}

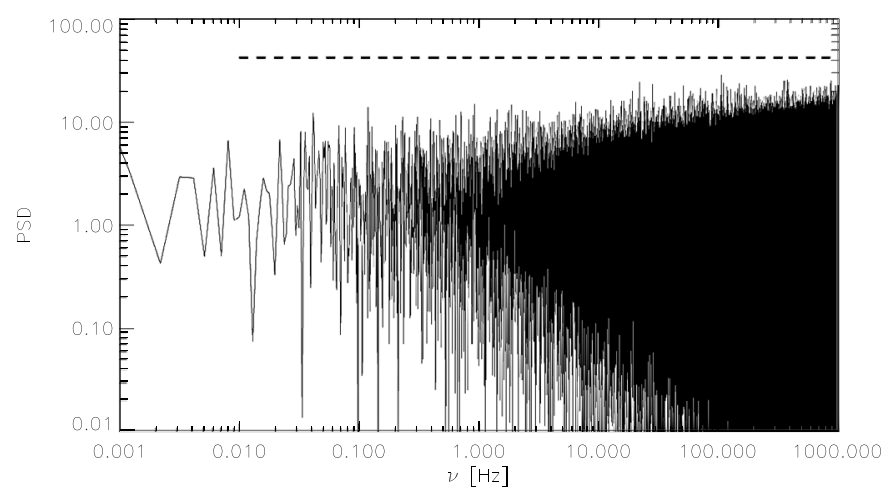

Fig. 2. Power spectral density obtained from the PCA events of PCU 1,2 , and 4 (we used a single time interval with bins of $0.49 \mathrm{~ms}$ ). The dashed line represents the $3 \sigma$ limit for detection of coherent signals.

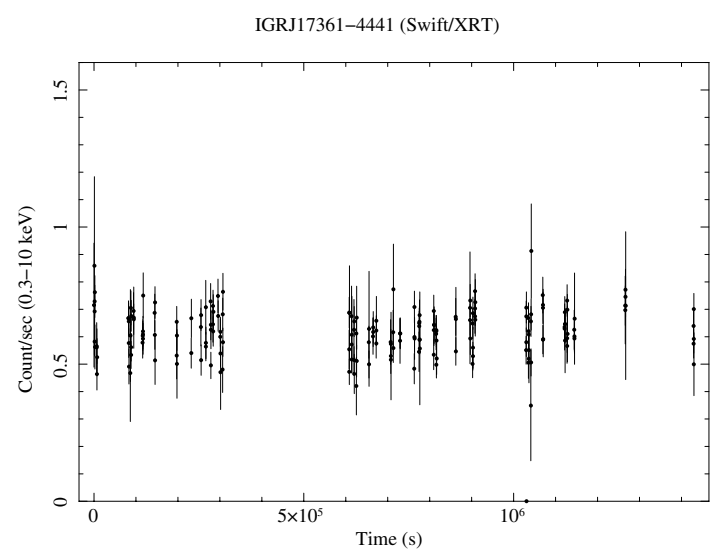

Fig. 3. Lightcurve of IGR J17361-4441 as observed by Swift/XRT (0.3$10 \mathrm{keV}$ ). The bin time is $250 \mathrm{~s}$ and the start time is 2011 August 16 at 17:01 (UTC).

\subsection{Swift}

Swift/XRT monitored the outburst of IGR J17361-4441 starting from 2011 August 16 for about 18 days (the last observation considered here was carried out on 2011 September 2 at 06:10 UTC). We processed all the Swift/XRT data with standard procedures (Burrows et al. 2005) and the latest calibration files available (caldb v. 20110725). All observations were performed in photon-counting mode ( $\mathrm{PC}$, time resolution $2.5 \mathrm{~s}$ ). The data analysis technique that we used is the same as that described in Bozzo et al. (2009). All the XRT spectra extracted from the available observations could be well accurately reproduced by using an absorbed power-law model with $N_{\mathrm{H}} \simeq$ $(0.2-0.5) \times 10^{22} \mathrm{~cm}^{-2}$ and $\Gamma \sim 0.5-0.9$. During this period, the source displayed a virtually constant X-ray flux of (4.5$4.8) \times 10^{-11} \mathrm{erg} / \mathrm{cm}^{2} / \mathrm{s}(1-10 \mathrm{keV}$ not corrected for absorption). The XRT lightcurve of the outburst is reported in Fig. 3. By using the Swift/XRT on-line analysis tool ${ }^{3}$ with all the XRT data available in the direction of NGC 6388, we also determined the new XRT position of IGR J17361-4441 to be RA = 17:36:17.27, Dec $=-44: 44: 07.0$, with an associated uncertainty of 1.9 arcsec (90\% c.1.; see Fig. 5). This is consistent with the position reported previously by Ferrigno et al. (2011b) and the refined Chandra position reported by Pooley et al. (2011). We studied the broad-band spectral properties of the X-ray emission

${ }^{3}$ See http://www.swift.ac.uk/user_objects/; see also Evans et al. (2009). 


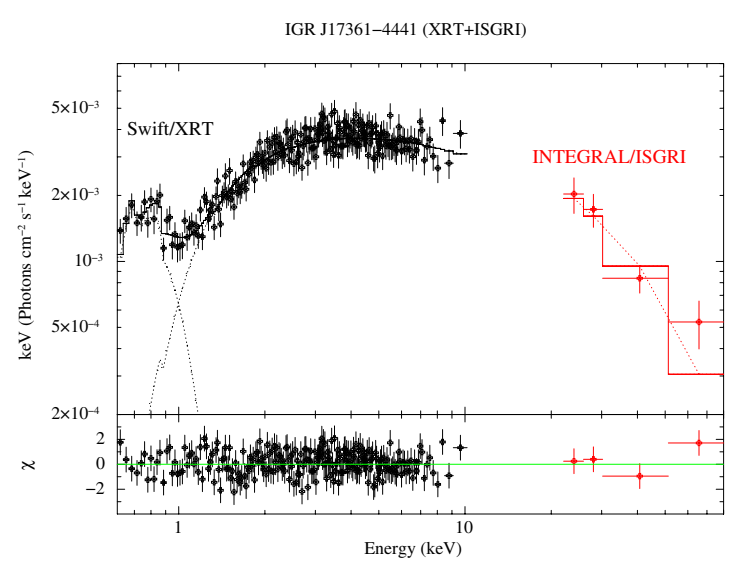

Fig. 4. Broad-band spectrum of IGR J17361-4441 obtained by using data from Swift/XRT and INTEGRAL/ISGRI. The best-fit model (absorbed cut-off PL plus a BB component, see text for details) and the residuals from this fit are also shown.

from IGR J17361-4441 by combining XRT data carried out before 2011 August 29 with those obtained during the same period with ISGRI (rev. 1078 to 1083). The joint XRT+ISGRI spectrum is shown in Fig. 4. We fit the two spectra together by using an absorbed power-law (PL) model with a cut-off at high energy (CUTOFFPL in XSPEC). A blackbody component (BB) was required to take into account the residuals at the lower energies $(\lesssim 2 \mathrm{keV})$. A normalization constant (fixed to 1 for the XRT spectrum) was included in the fit to account for the intercalibration between the different instruments and the variability (if any) of the source. From the fit $\left(\chi_{\text {red }}^{2} /\right.$ d.o.f. $\left.=0.95 / 269\right)$, we estimated that $N_{\mathrm{H}}=0.8 \pm 0.1 \mathrm{erg} / \mathrm{cm}^{2} / \mathrm{s}, \Gamma=1.0 \pm 0.1, E_{\text {cut }}=24_{-9}^{+20} \mathrm{keV}$, $k T_{\mathrm{BB}}=0.076 \pm 0.005 \mathrm{keV}$, and $R_{\mathrm{BB}}=1.4_{-0.5}^{+0.8} \times 10^{3} d_{10 \mathrm{kpc}} \mathrm{km}$ (where $E_{\text {cut }}$ is the cut-off energy, $d_{10 \mathrm{kpc}}$ is the source distance in units of $10 \mathrm{kpc}$, and all the uncertainties are given at the $90 \%$ c.l.). The normalization constant used for ISGRI turned out to be $C=1.2_{-0.5}^{+0.8}$ and no significant changes in the spectral fit parameters were observed by fixing $C=1$. The source average $\mathrm{X}$-ray flux was $\left(4.6_{-0.5}^{+0.1}\right) \times 10^{-11} \mathrm{erg} / \mathrm{cm}^{2} / \mathrm{s}$ in the $1-10 \mathrm{keV}$ energy band and $7.8_{-3.8}^{+0.8} \times 10^{-11} \mathrm{erg} / \mathrm{cm}^{2} / \mathrm{s}$ in the $20-100 \mathrm{keV}$ energy band. Compatible results were obtained by fitting the RXTE/PCA spectrum with the model described above. The $3-15 \mathrm{keV}$ X-ray flux measured from the PCA was $6.7_{-3.4}^{+0.1} \times 10^{-11} \mathrm{erg} / \mathrm{cm}^{2} / \mathrm{s}$, i.e. slightly higher than that extrapolated from the XRT energy band. Part of this discrepancy might be due to the limited time range spanned by the PCA data with respect to XRT and ISGRI, and possible contamination of the nearby bright persistent low mass X-ray binary 4U 1735-444 (located $\sim 0.5 \mathrm{deg}$ away from IGR J17361-4441, see also Sect. 2.2).

\subsection{ATCA}

To hel us establish the nature of IGR J17361-4441, we also obtained on 2011 August 25 a 8 h-long target of opportunity observation (ToO) with the Australia Telescope Compact Array (ATCA; Wilson et al. 2011) in configuration 6D (baseline is $6 \mathrm{~km}$ ). The observation was conducted with a $2 \mathrm{GHz}$ bandwidth centered around $5.5 \mathrm{GHz}, 9 \mathrm{GHz}, 17 \mathrm{GHz}$, and $19 \mathrm{GHz}$. The total integration time was about $5.3 \mathrm{~h}$ for the two lower frequencies (observed simultaneously) and $2.7 \mathrm{~h}$ for the higher frequencies (observed simultaneously). Natural-weighted images were made from the ATCA data, with FOVs (FWHM) of 8.5, 5.2, 2.8, and 2.5 arcmin, and synthesized beam sizes (FWHM) of $4.5 \times 1.8$,

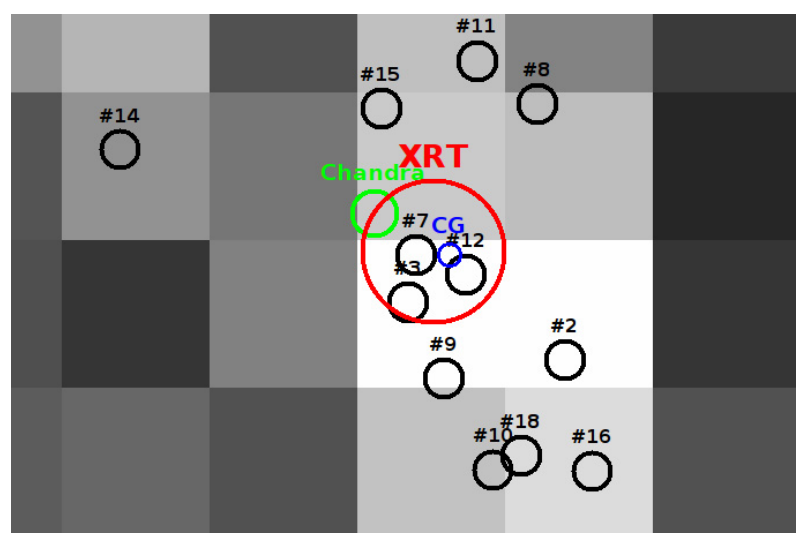

Fig. 5. Close view of the Chandra archival image (OBSID. 5055; 0.5$10 \mathrm{keV}$ ) around the core of NGC 6388. In the image, black circles correspond to all the Chandra sources detected so far in quiescence by Cseh et al. (position uncertainty 0.5 arcsec; 2010). The blue circle represents the COG of the GC (position uncertainty 0.3 arcsec). The improved XRT position determined in the present work is indicated with a red circle (see Sect. 2.3), and the Chandra position reported by Pooley et al. (2011) is represented with a green circle.

$2.7 \times 1.1,1.7 \times 0.6$, and $1.5 \times 0.5 \operatorname{arcsec}$ at $5.5,9,17$, and $19 \mathrm{GHz}$, respectively. No significant radio emission was found around the X-ray position of IGR J17361-4441. The rms noise levels of the images were $14.1 \mu \mathrm{Jy}, 19.0 \mu \mathrm{Jy}, 20.0 \mu \mathrm{Jy}$, and $21.2 \mu \mathrm{Jy}$ for the $5.5 \mathrm{GHz}, 9 \mathrm{GHz}, 17 \mathrm{GHz}$, and $19 \mathrm{GHz}$ frequencies, respectively. The rms noise level corresponding to the image in the $5.5 \mathrm{Ghz}$ frequency is a factor of $\sim 2.5$ lower than that reported previously by Cseh et al. (2010, see also Sect. 3).

\section{Discussion}

The X-ray monitoring campaign triggered after the discovery of IGR J17361-4441 with INTEGRAL, has enabled us to significantly improve the accuracy of the source position, and confirm its location within the core of the GC NGC 6388 (the COG of the GC has coordinates RA $=17: 36: 17.23$, Dec $=-44: 44: 07.1$; the estimated uncertainty is 0.3 arcsec; Lanzoni et al. 2007, see also Sect. 2.3). The XRT data have helped us to demonstrate that the source remained at a virtually constant flux during the first 18 days after the onset of the outburst, and did not display any significant spectral variability. We have been able to fit the broad-band $(0.5-100 \mathrm{keV})$ spectrum of the source reasonably well using a cut-off PL model $\left(N_{\mathrm{H}} \simeq 0.8 \times 10^{22} \mathrm{~cm}^{-2}\right.$, $\Gamma \simeq 0.7-1.0$, and $E_{\text {cut }} \simeq 25 \mathrm{keV}$ ) and displayed some hint of a soft spectral component below $\sim 2 \mathrm{keV}$. The estimated flux in the $1-100 \mathrm{keV}$ energy band is $1.7 \times 10^{-10} \mathrm{erg} / \mathrm{cm}^{2} / \mathrm{s}$, which corresponds to a luminosity of $L_{\mathrm{X}} \simeq 3.6 \times 10^{36} \mathrm{erg} / \mathrm{s}$ at the distance of NGC 6388 (13.2 kpc; Dalessandro et al. 2008).

The GC NGC 6388 was previously observed with Swift, XMM-Newton, and Chandra, because it is believed to host an IMBH in its COG (see also, Nucita et al. 2008, and references therein). To date, the deepest X-ray observations of the GC were carried out with Chandra and reported by Cseh et al. (2010). These authors found a number of sources located close to the core of the GC (sources \#3, \#7, and \#12), with spectral properties similar to those of the X-ray binaries ${ }^{4}$. and averaged quiescent luminosities of $L_{X} \simeq 10^{32}-10^{33} \mathrm{erg} / \mathrm{s}$. The relatively

${ }^{4}$ Source \#3 appeared to be rather soft and possibly compatible with being a cataclysmic variable. We do not discuss here this hypothesis for IGR J17361-4441 as its X-ray luminosity seems to be far too high with respect to that typically observed from these objects (see e.g., Heinke et al. 2008). 
small uncertainty in the position of IGR J17361-4441, as determined thanks to the Chandra observation of the source carried out by Pooley et al. (2011), suggests that the newly discovered INTEGRAL transient is associated with neither the objects detected previously in quiescence by Cseh et al. (2010), nor the COG of NGC 6388 (see Fig. 5). The quiescent luminosity of IGR J17361-4441 can thus be estimated at $\sim 10^{31} \mathrm{erg} / \mathrm{s}$ (Pooley et al. 2011), and is $\sim 10^{5}$ times lower than it was at outburst. The radio observation discussed in Sect. 2.4, showed that the large increase in the X-ray luminosity from IGR J17361-4441 in outburst was not followed by any re-brightening in the radio domain. If we were to use our results obtained at $5.5 \mathrm{GHz}$ and assume a distance to the source of $13.2 \mathrm{kpc}$, the rms level of 14.1 $\mu \mathrm{Jy}$ derived in Sect. 2.4 from the ATCA data would translate into a $3 \sigma$ upper limit on the radio luminosity from IGR J173614441 of $L_{\mathrm{R}}<5 \times 10^{28} \mathrm{erg} / \mathrm{s}$ (we adopted the same calculation procedure as in Cseh et al. 2010, see their Sect. 4). Assuming that the empirical relation between the mass of a $\mathrm{BH}$ and its radio and X-ray luminosity (the so-called "black-hole fundamental plane" Merloni et al. 2003; Körding et al. 2006) applies also in the present case, the measured values of $L_{X}$ and $L_{R}$ do not seem to be compatible with IGR J17361-4441 being a black-hole candidate (BHC) source, as the maximum allowed mass for this source would be far too low relative to expectations for these systems $\left(\gg M_{\odot}\right)$.

As the broad-band spectral properties of the source discussed in Sect. 2.3 are reminiscent of what is observed for some X-ray binaries hosting accreting neutron stars (NSXRBs; see e.g., Psaltis 2004, for a review), we propose that an alternative possibility is that IGR J17361-4441 belongs to this class of objects. Highly magnetized $\left(\sim 10^{12} \mathrm{G}\right)$ NSs in high mass X-ray binaries (HMXBs) usually display in outburst X-ray spectra comprising a relatively flat absorbed power-law $(\Gamma \simeq 0.5-1.0)$ component plus a cut-off at higher energies $(\sim 10-50 \mathrm{keV}$, sometimes related to the strength of the NS magnetic field; Coburn et al. 2002). Soft excesses ( $<2 \mathrm{keV})$ are also observed from these sources in both quiescence and outburst, which are ascribed to either an accretion disk or a diffuse cloud around the compact object (see e.g., Hickox et al. 2004). Alternatively, a hot spot on the star can also give rise to a BB component in the spectrum with typical $k T \sim 0.3-1 \mathrm{keV}$. Given the results suggested by the XRT data analysis $\left(R_{\mathrm{BB}} \simeq 1000 \mathrm{~km}\right)$, the first case would probably be the most likely one for IGR J17361-4441. However, at odds with typical HMXBs in outburst, the new transient source did not display any pronounced variability in the X-ray emission on time-scales of neither a few hundreds of seconds (as in wind accreting systems) nor days (as in disk accreting systems). The relatively young age of typical HMXBs (few $10^{6} \mathrm{yr}$ ) compared to that expected for the sources hosted in a globular cluster would also help us to exclude this hypothesis (the age of NGC 6388 has been estimated to be around $10^{10} \mathrm{yr}$; Moretti et al. 2009). For this reason, IGR J17361-4441 might be more easily associated with a low mass X-ray binary (ages $\gtrsim 10^{8} \mathrm{yr}$ ), even though these objects usually have softer spectra in the Xray domain $(\Gamma \sim 1.5-2)$. The lack of any pulsation in the RXTE data could also not be used to firmly establish the presence of a NS in IGR J17361-4441. As suggested by Wijnands et al. (2011), a different possibility is that IGR J17361-4441 is a very faint X-ray transient (VFXT; Wijnands et al. 2006; Campana $2009)$. These objects have similarly hard spectra $(\Gamma \sim 0.6-1)$, and might emit a relatively stable X-ray flux in outburst even for a few years (see, e.g., the case of XMMU J174716.1281048; Del Santo et al. 2007). However, these sources typically have a lower X-ray luminosity (in the range $10^{34}$-few $\times 10^{35} \mathrm{erg} / \mathrm{s}$ ) that that measured for IGR J17361-4441. Further observations of this new INTEGRAL transient with sensitive X-ray telescopes in the next few months might help us to understand the real nature of IGR J17361-4441.

Finally, we note that, as IGR J17361-4441 seems to be a new X-ray source in NGC 6388 and not associated with the IMBH possibly residing at its center, the ATCA upper limit derived in Sect. 2.4 gives a significantly tighter constraint of the allowed mass for this object with respect to those reported previously. Following the discussion in Cseh et al. (2010), we assume that the quiescent X-ray luminosity of the IMBH is that determined by these authors with Chandra and consider the updated $L_{\mathrm{R}}$ provided by our ATCA ToO observation. Inserting these values into Eq. (2) of Cseh et al. (2010) and taking into account the intrinsic scatter in the fundamental plane relation, the newly derived upper limit to the IMBH mass is $M<600 M_{\odot}$. This is a factor of 2.5 smaller than that reported by Cseh et al. (2010).

Acknowledgements. We thank the Swift, RXTE and ATCA teams for the rapid scheduling of the ToO observations of IGR J17361-4441, and an anonymous referee for useful comments. T.B. acknowledges financial support from ASI (INAFASI I/009/10/0). A.P. acknowledges the support of the operating program of Regione Sardegna (ESF 2007-2013), L.R.7/2007, "Promotion of scientific research and technological innovation in Sardinia". This research has made use of the IGR Sources page maintained by J. Rodriguez \& A. Bodaghee (http:// irfu.cea.fr/Sap/IGR-Sources/).

\section{References}

Bozzo, E., Giunta, A., Stella, L., et al. 2009, A\&A, 502, 21

Bradt, H. V., Rothschild, R. E., \& Swank, J. H. 1993, A\&AS (ISSN 0365-0138), 97,355

Burrows, D. N., Hill, J. E., Nousek, J. A., et al. 2005, Space Sci. Rev., 120, 165 Campana, S. 2009, ApJ, 699, 1144

Coburn, W., Heindl, W. A., Rothschild, R. E., et al. 2002, ApJ, 580, 394

Courvoisier, T., Walter, R., Beckmann, V., et al. 2003, A\&A, 411, L53

Cseh, D., Kaaret, P., Corbel, S., et al. 2010, MNRAS, 406, 1049

Dalessandro, E., Lanzoni, B., Ferraro, F. R., et al. 2008, ApJ, 677, 1069

Del Santo, M., Sidoli, L., Mereghetti, S., et al. 2007, A\&A, 468, L17

Evans, P. A., Beardmore, A. P., Page, K. L., et al. 2009, MNRAS, 397, 1177

Ferrigno, C., Bozzo, E., Falanga, M., et al. 2011a, A\&A, 525, A48

Ferrigno, C., Bozzo, E., Rodriguez, J., \& Gibaud, L. 2011b, The Astronomer's Telegram, 3566, 1

Gibaud, L., Bazzano, A., Bozzo, E., et al. 2011, The Astronomer's Telegram, 3565,1

Heinke, C. O., Ruiter, A. J., Muno, M. P., \& Belczynski, K. 2008, in A Population Explosion: The Nature \& Evolution of X-ray Binaries in Diverse Environments, ed. R. M. Bandyopadhyay, S. Wachter, D. Gelino, \& C. R. Gelino, AIP Conf. Ser., 1010, 136

Hickox, R. C., Narayan, R., \& Kallman, T. R. 2004, ApJ, 614, 881

Körding, E., Falcke, H., \& Corbel, S. 2006, A\&A, 456, 439

Lanzoni, B., Dalessandro, E., Ferraro, F. R., et al. 2007, ApJ, 668, L139

Lebrun, F., Leray, J. P., Lavocat, P., et al. 2003, A\&A, 411, L141

Lund, N., Budtz-Jørgensen, C., Westergaard, N. J., et al. 2003, A\&A, 411, L231

Merloni, A., Heinz, S., \& di Matteo, T. 2003, MNRAS, 345, 1057

Moretti, A., Piotto, G., Arcidiacono, C., et al. 2009, A\&A, 493, 539

Nucita, A. A., de Paolis, F., Ingrosso, G., Carpano, S., \& Guainazzi, M. 2008, A\&A, 478, 763

Pooley, D., Homan, J., Heinke, C. O., et al. 2011, The Astronomer's Telegram, 3627,1

Psaltis, D. 2004 [arXiv: astro-ph/0410536]

Ubertini, P., Lebrun, F., Di Cocco, G., et al. 2003, A\&A, 411, L131

Vaughan, B. A., van der Klis, M., Wood, K. S., et al. 1994, ApJ, 435, 362

Wijnands, R., in't Zand, J. J. M., Rupen, M., et al. 2006, A\&A, 449, 1117

Wijnands, R., Yang, Y., Degenaar, N., et al. 2011, The Astronomer's Telegram, 3595, 1

Wilson, W. E., Ferris, R. H., Axtens, P., et al. 2011, MNRAS, accepted [arXiv: 1105.3532$]$

Winkler, C., Courvoisier, T., Di Cocco, G., et al. 2003, A\&A, 411, L1 